\title{
Esthetic Gingival Management using Combination of Platelet-rich Fibrin and Collagen membrane: A Case Report
}

\author{
Varun Choudhary, Manab Kosala, S. K. Bhandari \\ Division of Periodontology, Department of Dental Surgery and Oral Health Sciences, AFMC, Pune, Maharashtra, India
}

Email for correspondence: drvarunchoudhary@gmail.com

\begin{abstract}
Gingival recession can result in root sensitivity, esthetic concern to the patient, and predilection to root caries. Esthetic treatment such as root coverage procedures has become integral part of periodontal treatment. Platelet-rich fibrin (PRF) is an autogenous, less invasive and effective mode of treating gingival recession. The aim of the study was to assess the clinical outcome following use of PRF and collagen membrane along with coronally advanced flap to manage Miller Class I gingival recession defects in maxillary anterior region. PRF can be used as a membrane for periodontal tissue regeneration due to the ability to promote platelet aggregation, chemotactic for fibroblast and enhance wound stability, and proper healing. Hence, PRF in combination with collagen membrane can be successfully used as alternative to connective tissue graft for treating gingival recession.
\end{abstract}

Key words: Collagen membrane, coronally advanced flap, platelet-rich fibrin

\section{INTRODUCTION}

Gingival recession is an apical migration of the gingival margin tissue relative to the cementoenamel junction (CEJ) characterized by the exposure of the root surface. Several factors influence the development of marginal tissue recession including traumatic tooth brushing technique trauma, plaqueinducedinflammation, tooth alignment, orthodontics, restorative procedures, high muscle attachment, and frenal pull..$^{[1,2]}$ Isolated gingival recessions have been treated by several techniques. ${ }^{[3]}$ The migration of the marginal tissue in an apical direction may lead to esthetic, dentin hypersensitivity, root caries, and cervical wear recessions. Various methods for performing mucogingival grafts to cover denuded root surfaces are available. However, coronally advanced flap $(\mathrm{CAF})$ is a predictable surgical procedure when

\section{Quick Response Code Article Info:}

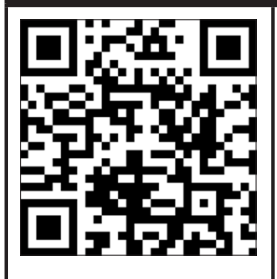

doi: $10.5866 / 2018.10 .10187$

Received: $28-09-2018$

Revised: $27-10-2018$

Accepted: 19-11-2018

Available Online: 05-01-2019, (www.

nacd.in)( NAD, 2019 - All rights reserved there is adequate keratinized tissue present apical to the recession defect in the treatment of Miller Class I and II gingival recessions. ${ }^{[4]} \mathrm{CAF}$ alone gives an unstable result for long-term period, despite of having many advantages. Such procedure leads to formation of long junctional epithelium, which is risk factor for future recurrence of gingival recession.

To improve the results, various regenerative procedures have been tried with CAF which enhances root coverage. One of such material is platelet-rich fibrin (PRF) which is a secondgeneration autologous platelet concentrate and does not require any extensive preparation as in need in the preparation of platelet-rich plasma (PRP). The growth factors within platelet concentrates (PRP and PRF) upregulate cellular activity, effective in the enhancement of early wound healing and are promoters of periodontal tissue regeneration. ${ }^{[5]}$ $\mathrm{PRF}$ is a 3-D polymerized matrix in which platelet, cytokines, leukocytes, and various growth factors including vascular endothelial growth factor, insulin-like growth factor, platelet-derived growth factor, transforming growth factor, epidermal growth factor, basic fibroblast growth factor, and circulating stem cells are trapped and that can 
serve as a resorbable membrane. Due to these factors, PRF accelerates wound healing and has a supportive effect on the immune system, cell migration, and proliferation. ${ }^{[6,7]}$ At the same time, early retraction nature of PRF which can make it less stable and hindered its efficacy collagen membrane (HEALIGUIDE ${ }^{\circledR}$ ) was used as a carrier material. Due to various inherent factors present in autologous platelet concentrate which has the capacity for bone regeneration and accelerates soft tissue wound healing, the gingival recession shown in the present case report was treated using autologous PRF and collagen membrane along with CAF.

\section{CASE REPORT}

A 35-year-old male patient reported with a chief complaint of sensitivity in upper front teeth. No associated medical history was present. Enquiry regarding oral hygiene practices disclosed improper brushing habit. Intraoral examination revealed Miller's Class I gingival recession in relation to 13 measuring $3 \mathrm{~mm}$ [Figure 1]. Probing depth was recorded as $3 \mathrm{~mm}$ around involved tooth. The width of keratinized gingiva was found to be in normal range.

\section{Pre-surgical Procedure}

The surgical procedure was explained to the patient and informed consent was obtained. Preparation of the patient was carried out, which included scaling and root planning of the entire dentition and oral hygiene instructions. The probing depth was recorded as $2 \mathrm{~mm}$ after initial preparation.

\section{PRF Preparation}

The PRF was prepared following the protocol developed by Choukroun et al. ${ }^{[8]}$ Before surgery, intravenous blood was drawn from antecubital vein in 10-ml sterile tubes and immediately centrifuged in centrifugation machine at 3000 revolutions per minute for $10 \mathrm{~min}$. At the end of centrifugation, three layers were seen, the top layer containing supernatant serum, the fibrin clot at the middle layer, and the bottom layer containing the red blood corpuscles [Figure 2]. With the help of sterile tweezers and scissors, the fibrin clot was easily separated from the RBC base and then kept in a sterile Dappen Dish [Figure 3]. Before use, it was slightly squeezed between the gauze pieces to remove its serum content.

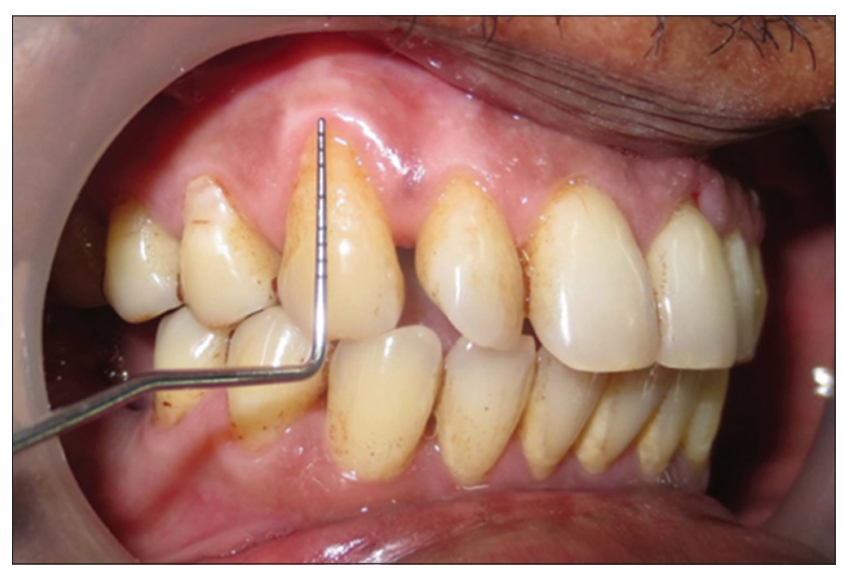

Figure 1: Clinical picture showing Miller's Class I gingival recession in relation to 13 measuring about $3 \mathrm{~mm}$

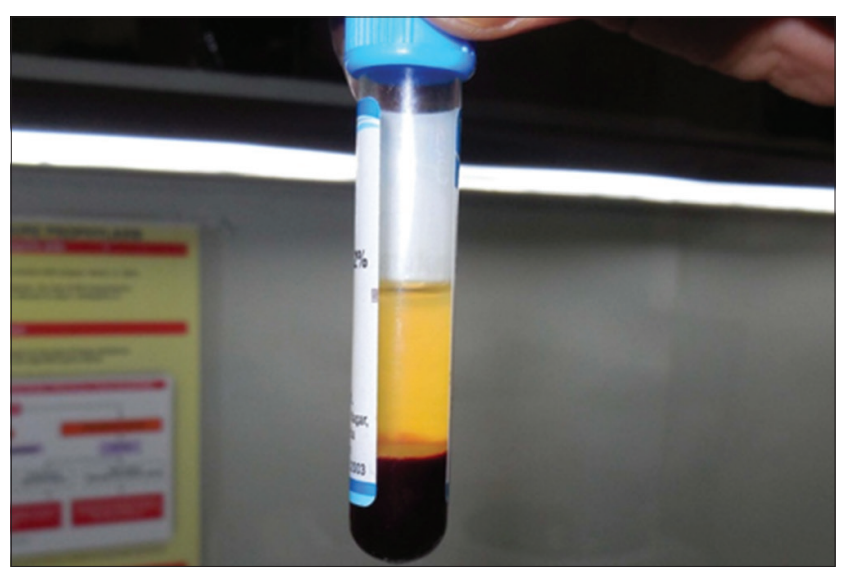

Figure 2: Platelet-rich fibrin preparation showing three layers, the top layer containing supernatant serum, the fibrin clot at the middle layer, and the bottom layer containing the red blood corpuscles

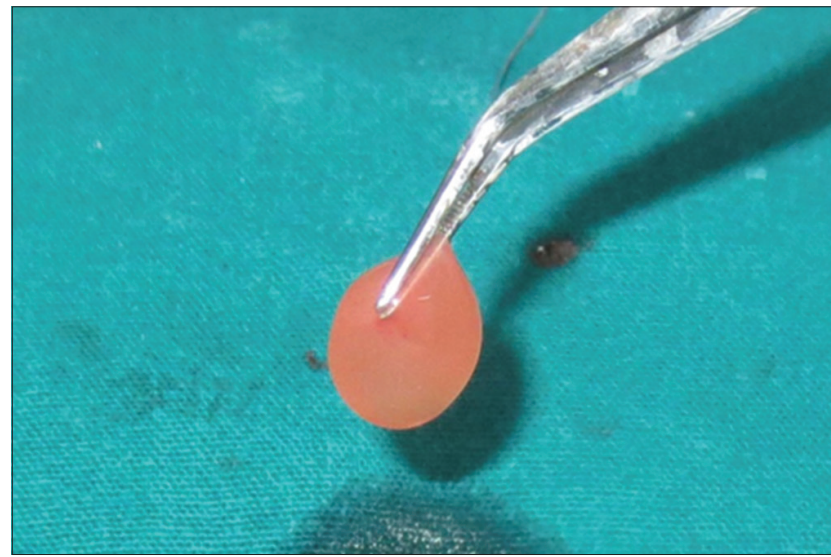

Figure 3: Separation of fibrin clot from the red blood cell base with the help of sterile tweezers 


\section{Surgical Procedure}

After giving local anesthesia (1:80,000 adrenaline), twohorizontalincisions were madeonboth sides parallel to the CEJ of the tooth to be treated with a no. $15 \mathrm{C}$ blade, creating a well-defined butt joint margin. In this way, interdental papillae can be preserved for a better outcome. Vertical releasing incisions were made on the mesial and the distal aspects at the surgical site (at the terminal ends of the horizontal incisions) and placed at the line angles of adjacent teeth. These releasing incisions were extended beyond the mucogingival junction for the flap to be mobile. An intrasulcular incision around the affected tooth was made that connected the two vertical incisions coronally with the horizontal incisions [Figure 4].

A full-thickness flap was raised using a periosteal elevator (24G) (Hu-Friedy) which enabled

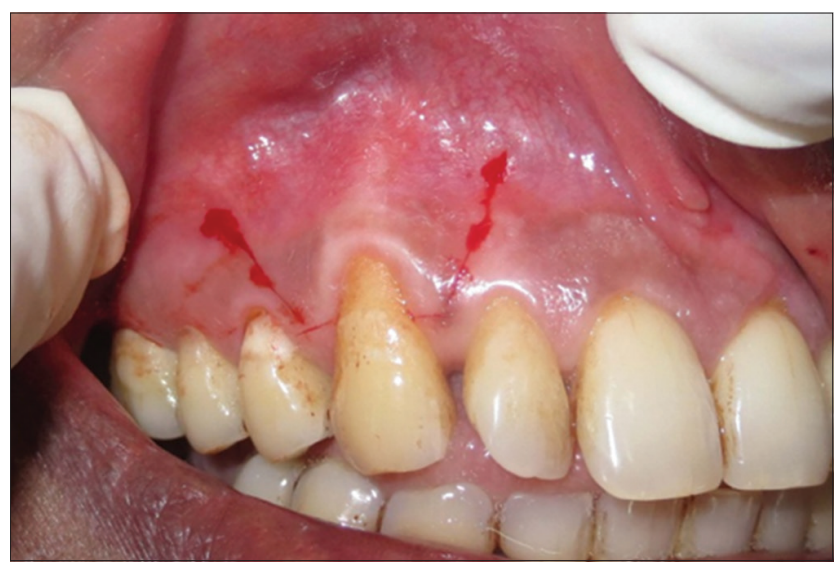

Figure 4: Clinical picture showing the placement of intrasulcular incision around the affected tooth that connects the two vertical incisions coronally with the horizontal incisions

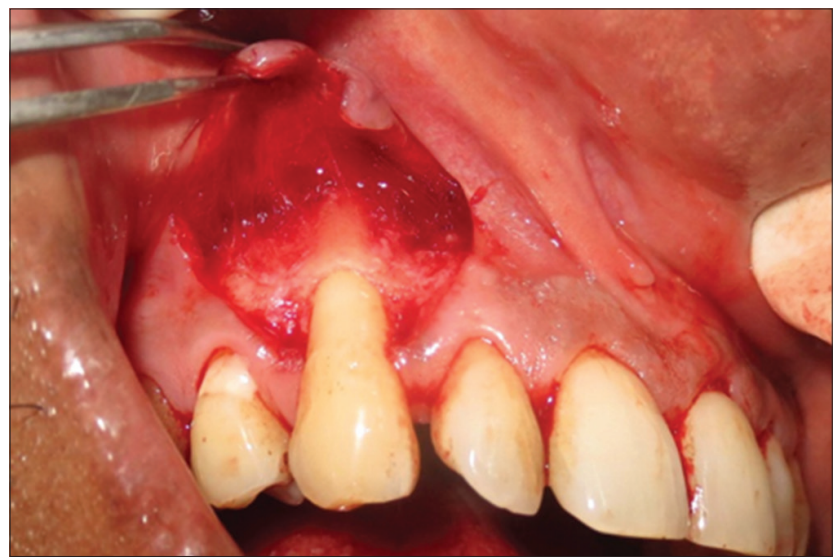

Figure 5: Clinical picture showing raising of full-thickness flap that enabled bone visibility of $3 \mathrm{~mm}$ apical to the exposed root bone visibility of $3 \mathrm{~mm}$ apical to the exposed root [Figure 5]. The flap was then converted to a partial thickness apically that will enable coronal mobilization. When moved coronally, the falp was now able to cover and lie passively on the recession area without any tension. This is critical because any tension while suturing will affect the positive outcome of the procedure. The collagen membrane was trimmed to cover the recession area properly. It was adjusted in such a way that it extended $2 \mathrm{~mm}$ beyond the borders of the recession mesially, distally, and apically. PRF in a collagen membrane carrier was placed coronally at the level of CEJ and was secured [Figure 6]. Once the PRF and membrane are stabilized, then the buccal flap was moved coronally and secured to the papillae with interrupted suture using 4-0 absorbable sutures [Figure 7]. Gentle pressure was applied at the surgical site with moistened gauze to achieve hemostasis and followed

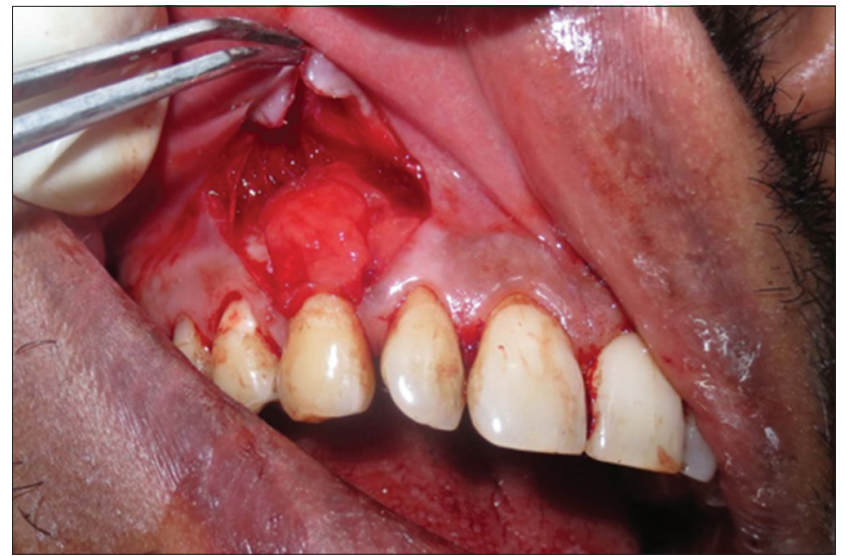

Figure 6: Placement of platelet-rich fibrin in a collagen membrane and was secured

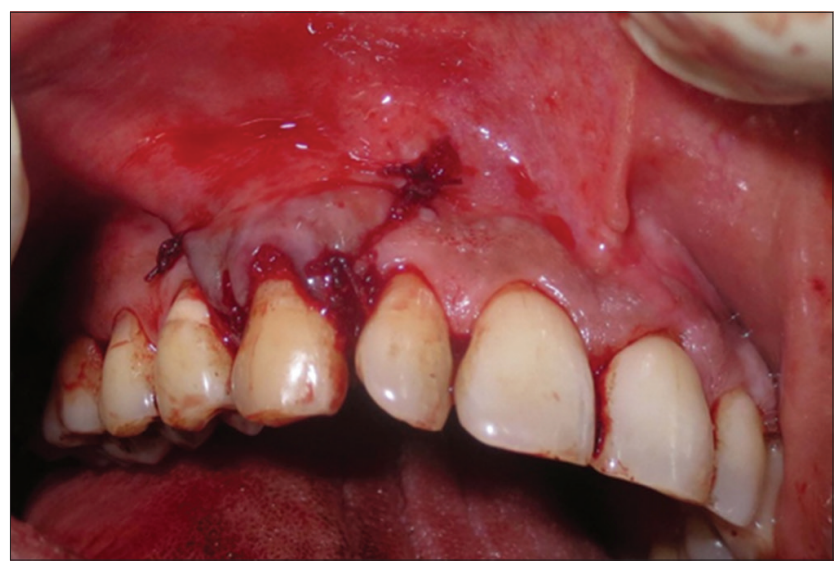

Figure 7: Placement of sutures 


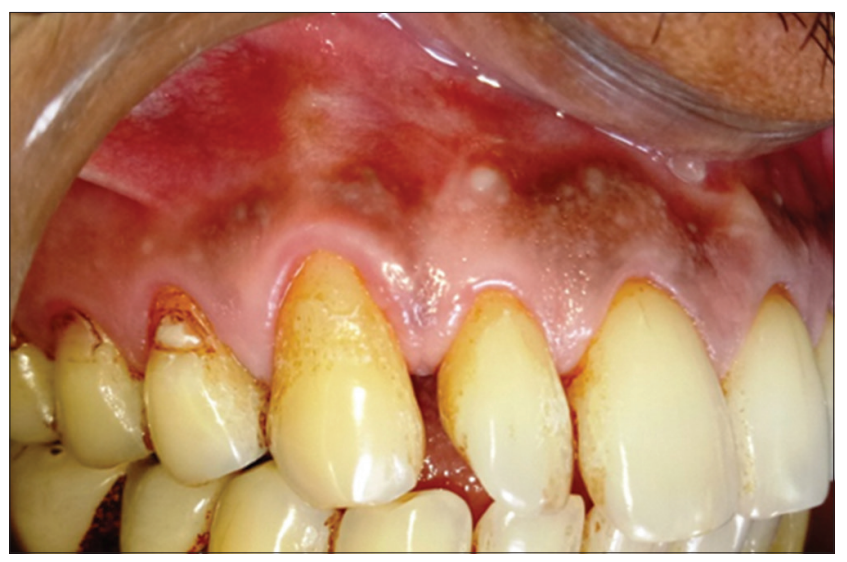

Figure 8: Follow-up at 1 year showing $100 \%$ root coverage

by the periodontal dressing. A periodontal dressing was then placed on the surgical site and left for a week.

\section{Post-surgical Care}

Patients were prescribed with antibiotics and analgesics (Cap. Amoxicillin 500 mg, TDS and Tab. Paracetamol + Ibuprofen, and TDS for 3 days). Post-operative instructions were given and the patient was informed to report after 10 days for suture removal. Complete root coverage was noticed at that time. Professional scaling and oral hygiene reinforcement were provided at each follow-up visit whenever indicated. Follow-up recorded 1 year postoperatively was shown $100 \%$ root coverage [Figure 8].

\section{DISCUSSION}

The correction of the gingival recession has become highly critical due to high esthetic demand and other patient-centered complaints such as hypersensitivity, risk of root caries, pulpal symptoms due to root exposure, food lodgement, and plaque deposition in the developmental grooves. The decision of selection of a particular technique over the array of modalities requires careful evaluation of various patient-related and surgical variables. The gold standard grafting technique in recession coverage is connective tissue grafting (CTG) with the good color match and predictable outcome. ${ }^{[9]}$ Nevertheless, it is also associated with complications such as increased patient morbidity and limited tissue availability. Guided tissue regenerative (GTR) as a regenerative option for recession coverage is promising, with no statistical difference in the treatment outcome when compared with that of CTG. ${ }^{[10]}$ The use of collagen membrane as a resorbable barrier aids in the maintenance of principles of GTR, namely space provision, stability, compartmentalization, and angiogenesis. PRF as an alternative soft tissue grafting material has various advantages over the CTG. A study comparing CTG and PRF has concluded that the PRF group exhibited early vascularization in the wound. ${ }^{[11]}$ Its usefulness in recession management has been ascertained in the dental literature. ${ }^{[12]}$ Thus, PRF is an ideal bio-healing material with positive wound healing response in the gingival recession management. In addition, the favorable results of the studies on GTR with the application of platelet concentrate in the treatment of infrabony defects also echoed the laboratory findings. ${ }^{[13,14]}$

However, at the same time, one disadvantage associated with PRF that it rapidly resorbs in a few days after its placement and it is only partially able to maintain a space and a barrier against epithelial cells penetration. In the present case report, at 1 year, $100 \%$ mean root coverage was noted, with complete coverage in relation to 13 . Furthermore, the uncomplicated wound healing reflects the wound healing potential of PRF. Thus, CAF with PRF and collagen membrane with their tissue healing properties gives an added benefit in tackling these confounding defects. Therefore, the association of collagen membrane and PRF could be a good idea to obtain good tissue regeneration in less time and with a great healing of soft tissues. No histologic evaluation was performed to assess the type of healing. Therefore, the effect of PRF in association with collagen membrane on the establishment of a connective tissue attachment remains to be determined. Further evaluation is necessary to find out the type of healing, histologically as well as long-term follow-up of the clinical case.

\section{CONCLUSION}

Obtaining predictable and esthetic root coverage are the goal of periodontal plastic surgery. The current case report demonstrates the advantage of using CAF with the growth factors presence of PRF and biomatrix of collagen membrane in treating gingival recession with superior wound healing, satisfactory patient esthetics and improved functional result.

\section{REFERENCES}

1. Checchi L, Daprile G, Gatto MR, Pelliccioni GA. Gingival recession and toothbrushing in an Italian school of dentistry: A pilot study. J Clin Periodontol 1999;26:276-80. 
2. Trott JR, Love B. An analysis of localized gingival recession in 766 Winnipeg High School students. Dent Pract Dent Rec 1966;16:209-13.

3. Roccuzzo M, Bunino M, Needleman I, Sanz M. Periodontal plastic surgery for treatment of localized gingival recessions: A systematic review. J Clin Periodontol 2002;29 Suppl 3:178-94.

4. Baldi C, Pini-Prato GP, Pagliaro U, Nieri M, Saletta D, Muzzi L, et al. Coronally advanced flap procedure for root coverage. Is flap thickness a relevant predictor to achieve root coverage? A 19 case series. J Periodontol 1999;70:1077-84.

5. Del Corso M, Vervelle A, Simonpieri A, Jimbo R, Inchingolo F, Sammartino G, et al. Current knowledge and perspectives for the use of platelet-rich plasma (PRP) and platelet-rich fibrin (PRF) in oral and maxillofacial surgery part 1: Periodontal and dentoalveolar surgery. Curr Pharm Biotechnol 2012;13:1207-30.

6. Ehrenfest DM, Bielecki T, Del Corso M, Inchingolo F, Sammartino G. Shedding light in the controversial terminology for platelet-rich products: Platelet-rich plasma (PRP), platelet-rich fibrin (PRF), platelet-leukocyte gel (PLG), preparation rich in growth factors (PRGF), classification and commercialism. J Biomed Mater Res A 2010;95:1280-2.

7. Ehrenfest DM, Diss A, Odin G, Doglioli P, Hippolyte MP, Charrier JB. In vitro effects of Choukroun's PRF (plateletrich fibrin) on human gingival fibroblasts, dermal prekeratinocytes, preadipocytes, and maxillofacial osteoblasts in primary cultures. Oral Surg Oral Med Oral Pathol Oral Radiol Endod 2009;108:341-52.
8. Choukroun J, Diss A, Simonpieri A, Girard MO Schoeffler C, Dohan SL, et al. Platelet-rich fibrin (PRF) A second-generation platelet concentrate. Part IV: Clinical effects on tissue healing. Oral Surg Oral Med Oral Pathol Oral Radiol Endod 2006;101:56-60.

9. Dembowska E, Drozdzik A. Subepithelial connective tissue graft in the treatment of multiple recession. Oral Surg Oral Med Oral Pathol Oral Radiol Endod 2007;104;1-7.

10. Tatakis DN, Trombelli L. Gingival recession treatment: Guided tissue regeneration with bioabsorbable membrane versus connective tissue graft. J Periodontol 2000;71:299-307.

11. Eren G, Kantarcı A, Sculean A, Atilla G. Vascularization after treatment of gingival recession defects with plateletrich fibrin or connective tissue graft. Clin Oral Investig 2016;20:2045-53.

12. Aleksi Z, Jankovi S, Dimitrijevi B, Divni -Resnik T, Milinkovi I, Lekovi V. The use of platelet-rich fibrin membrane in gingival recession treatment. Srp Arh Celok Lek 2010;138:11-8.

13. Camargo PM, Lekovic V, Weinlaender M, Vasilic $\mathrm{N}$, Madzarevic M, Kenney EB. Platelet-rich plasma and bovine porous bone mineral combined with guided tissue regeneration in the treatment of intrabony defects in humans. J Periodontal Res 2002;37:300-6.

14. Lekovic V, Camargo PM, Weinlaender M, Vasilic N, Kenney EB. Comparison of platelet-rich plasma, bovine porous bone mineral, and guided tissue regeneration versus platelet-rich plasma and bovine porous bone mineral in the treatment of intrabony defects: A reentry study. J Periodontol 2002;73:198-205. 